\title{
Microbiological and Immunological Aspects of Narcolepsy
}

\author{
Demeke Geremew ${ }^{1,2}$, Meseret Workineh ${ }^{1}$, Maryam Izad ${ }^{2, *}$ \\ ${ }^{1}$ Department of Immunology and Molecular Biology, School of Biomedical and Laboratory Sciences, \\ University of Gondar (UoG), Ethiopia \\ ${ }^{2}$ Department of Immunology, School of Medicine, Tehran University of Medical Sciences International Campus (TUMS-IC), Iran
}

Copyright $\subset 2017$ by authors, all rights reserved. Authors agree that this article remains permanently open access under the terms of the Creative Commons Attribution License 4.0 International License

\begin{abstract}
Narcolepsy is a chronic neurological disorder characterized by excessive day time sleepiness, cataplexy and hypnagogic and hypnopompic hallucination. Narcolepsy is caused by loss of hypocretine neuron. Hypocretine is an excitatory neuropeptide projecting in the central nervous system and maintains wakefulness. The mechanism of hypocretin loss in narcolepsy is elusive. However, an autoimmune mediated destruction of hypocretin neuron is suspected because of its firm association with HLA-DQB1*0602 genotype and polymorphism in the TCR- $\alpha$ chain. Furthermore, infection with streptococcus pyogenes, influenza A H1N1 strain and the Pandemrix vaccine itself has strong association with narcolepsy. We searched PubMed (1984 - 2015), Google Scholar (1984 2015), MEDLINE (1984 - 2015), and EMBASE (1984 2015). In the interim, bibliographies of identified articles were reviewed to find additional references. The purpose of this mini review is to summarize recent findings involving microbiological and immunological factors for narcolepsy development.
\end{abstract}

Keywords Narcolepsy, Cataplexy, Hypocretin, HLA-DQB $1 * 0602$, Pandemrix Vaccine

\section{Introduction}

Narcolepsy is a chronic neurological disorder caused by loss of hypocretine (Hcrt) neurons, wakefulness associated neurotransmitter in the lateral hypothalamus [1, 2]. The clinical features of narcolepsy includes: excessive daytime sleepiness, cataplexy, hypnagogic hallucinations, sleep paralysis, and disturbed nocturnal sleep patterns [1]. The International Classification of Sleep Disorders (ICSD-3) classified narcolepsy in to two main forms: type $1 \&$ type 2 narcolepsy [3]. A typical feature of type 1 narcolepsy is sudden loss of muscle tone triggered by positive emotions [4, 5] such as laughter and surprise [5-9]. The usual onset of the disease is in childhood,with highest incidence between 10 19 age groups [3], and once established the disease is life-long $[10,11]$. Narcolepsy affects $0.03-0.16 \%$ of the general population in various ethnic groups [12], and the highest prevalence $(0.16 \%)$ was reported from Japan [13]. In Europe, the average direct and indirect costs of narcolepsy were estimated to be $€ 3200-18000$ and $€ 1000-11000$ per case per year respectively [3].

Genetic and environmental factors play key roles for the development of narcolepsy [12, 14]. Among the different genetic predisposing factors, a specific human leukocyte antigen (HLA) subtype is the major trigger [15]. Furthermore, environmental association with upper airway infections; streptococcus pyogenes, and influenza A virus $\mathrm{H} 1 \mathrm{~N} 1$ vaccination and infection itself was reported to have an important effect on narcolepsy susceptibility [16-19]. Recent pathophysiological studies have shown that type 1 narcolepsy is caused by the early loss of Hcrt, but type 2 narcolepsy is rarely caused by Hcrt deficiency [20] indicating a more heterogeneous origin in its etiology [21]. In this review, we will discuss the main genetic and environmental triggers associated with microbiology and immunology, which leading to an autoimmune mediated destruction of Hcrt neurons in narcolepsy.

\section{Causes of Narcolepsy}

\subsection{Genetic Triggers to Narcolepsy}

In 1984, Juji and colleagues discovered a strong association of narcolepsy with HLA class II region [22]. Subsequently, Mignot et al. uncovered that HLA-DQ0602, a heterodimer protein encoded by HLA-DQA1*01:02 \& DQB1*06:02 as the main predisposing genes [23, 24]. Moreover, other HLA alleles such as DQB1*03:01 also contribute to narcolepsy susceptibility, but the effect is weaker [3]. Nevertheless, HLA-DQB1 *06:01, DQB1*05:01, DQA1*01(non- DQA1*01:02) and DQB1*06:03 are protective alleles to narcolepsy $[3,25]$.

Type 1 narcolepsy is strongly associated with HLA-DQ0602, especially with DQB1*06:02 (90-100\%), the overwhelming effect of this allele on the risk suggests that 
HLA- DQ0602 present antigenic peptides to $\mathrm{CD}^{+} \mathrm{T}$ cells [26-29]. However, only $20-30 \%$ of patients with type 2 narcolepsy are positive for DQB1*06:02 allele [20, 21]. Most notably, DQB1*06:02 homozygosity increases narcolepsy susceptibility by two to four times than heterozygotes [25, 30, 31]. Nevertheless, expression of DQB1*06:02 is not limited to narcoleptic patients; rather 12 $-38 \%$ of the general population are carriers of this allele $[2,8$, $9,32,33]$, but very few carriers of this relatively common allele develop narcolepsy, indicating the involvement of other genetic or environmental factors [34].

Genome wide association studies (GWAS) of individuals of European ancestry have identified other genetic triggers to narcolepsy that includes; single nucleotide polymorphisms (SNPs) of the genes encoding T-cell-receptor $\alpha$ chain (TCR- $\alpha$ ) $[2,4,8,18,26,35-38]$, Purinergic receptor subtype P2RY11 [2, 8, 17, 18, 25, 26, 35, 38-40], Tumor necrosis factor (ligand) superfamily member 4 (TNFSF4), also called OX40L $[2,8,18,26]$, Cathepsin H (CTSH) $[2,8,18,26]$ and DNA methyltransferase-1 (DNMT1) $[2,8,18,26,31]$. The involvement of specific variants in the TCR alpha locus of $\mathrm{J}$ region segment and DQB1*06:02 in narcolepsy strongly suggest that it is a $\mathrm{T}$ cell-mediated autoimmune diseases [2, 8]. The autoimmune nature of narcolepsy in HLA-DQB1*0602 positive patients is explained by excessive lymphocyte reactivity and loss of natural tolerance to slightly modified antigens after a viral or bacterial infection [41].

\subsection{The Role of Upper Airway Infections in Narcolepsy}

In genetically susceptible individuals, various types of autoimmune disorders affecting neurons in the CNS as a result of environmental triggers have been reported $[8,10]$. The role of environmental factors in the development and progression of narcolepsy is clearly apparent when considering the low disease concordance rate between monozygotic twins [4, 14, 42-44]. Of all environmental factors, upper airway infections specifically streptococcus pyogenes and influenza A H1N1 infection and selected $\mathrm{H} 1 \mathrm{~N} 1$ vaccine preparations was strongly correlated to the onset of the disease in children and young adults $[1,10,31$, 45, 46, 47]. Namely, AS03-adjuvanted vaccine like Pandemrix (a vaccine with squalene and alpha tocopherol mix adjuvant) was the main trigger to narcolepsy $[1,10,31$, 46, 47]. As infectious disorders commonly show seasonal variation [3], the finding is also supported by the robust seasonality of the disease onset $[11,17,48]$, suggesting that winter H1N1 infections could trigger narcolepsy [17, 47].

Among various mechanisms by which host infection by a pathogen can lead to autoimmunity [1, 49], bystander activation and molecular mimicry are the major means by which upper airway infections may induce an autoimmune destruction of hypocretin neurons in the CNS of narcoleptic patients $[1-3,17,42,50,51]$.

\subsubsection{Streptococcus Pyogenes Superantigens and Narcolepsy}

Genetically susceptible individuals develop narcolepsy as a complication of a streptococcal infection [52]. The risk of narcolepsy in patients with a physician diagnosed streptococcal infection was estimated to be 5.4 times higher than in individuals without such a history [12, 17, 52, 53]. Moreover, the involvement of streptococcal infections in the development of the disease was further supported by the presence of anti-streptococcal antibodies, anti-streptolysin $\mathrm{O}$ (ASO), in narcoleptic patients [8, 17, 31, 48, 53, 54].

Streptococcus pyogenes superantigens (SAgs) bind primarily to the beta chain of the TCR and the alpha chain of $\mathrm{MHC}$ and generate polyclonal $\mathrm{T}$ cell responses [8]. However, there are also known SAgs that bind to alpha chains of the TCR, and cause bystander activation or increased permeability of the blood brain barrier (BBB) to auto reactive $\mathrm{T}$ cells, thus, it might be possible that the observed narcolepsy associated TCR alpha polymorphism may reflect involvement of streptococcal SAgs in narcolepsy [17]. Moreover, Streptococcal infections can induce the production of IL-17, and IL-17 receptor signaling has been shown to facilitate permeability of the BBB, potentially exposing hypocretin secreting neurons to the immune system $[2]$.

\subsubsection{Influenza A H1N1 Strain or Pandemrix Vaccine and Narcolepsy}

With the declaration of a global pandemic influenza A H1N1 virus by World Health Organization (WHO) in June 2009, mass vaccination campaigns using newly developed monovalent vaccines were initiated in a number of countries using a range of vaccines developed with different technologies [55, 56]. Thereafter, postmarketing safety assessments in Scandinavian and European countries have reported an increased risk of narcolepsy among children and young adult receipts of AS03-adjuvanted vaccine [7, 40, 55, 57-59]. In Finland, it was reported that AS03-adjuvanted vaccine increased the risk of narcolepsy by 13 fold in genetically susceptible children and young people $[3,4,46$, 58-60]. However, why other H1N1 vaccines, such as those with squalene alone as an adjuvant (MF59 adjuvanted) or non adjuvanted vaccine did not strongly associated to narcolepsy incidence remains unclear [2].

A study from China showed a clear seasonal variation in narcolepsy incidence, which increased after the 2009 H1N1 pandemic indicating that not only vaccination but also the influenza virus infection per se could trigger narcolepsy $[4,8$, $47,48]$. Incidence of childhood narcolepsy was increased 3 fold following the winter H1N1 infections of 2009-2010, independent of vaccination, suggesting that H1N1 infections by itself increase susceptibility [17, 48].

The pHA1 ${ }_{275-287}$ epitope specific to haemaglutinin 1 in the 2009 H1N1 strain or AS03 adjuvanted vaccine is similar to the hypocretin antigenic epitopes Hcrt56-68 and Hcrt87-99, this molecular similarity was noted in the amino 
acids important for the peptide binding to the DQ0602 allele, and this resulted in cross-reactive $\mathrm{CD} 4^{+} \mathrm{T}$ cell responses not only to pHA1 $275-287$ epitope, but also to Hcrt56-68 and Hcrt87-99 antigenic epitopes [3, 47]. Such sequence similarities were initially identified by homology searches, and more recently with search algorithms that consider the structural requirements for TCR recognition of MHC-bound peptides [61]. Therefore, Molecular mimicry due to structural features of MHC-peptide-TCR complexes that limit TCR specificity for MHC-bound peptides could cause destruction of Hcrt epitopes by cross reactive $\mathrm{CD}^{+} \mathrm{T}$ cells presented by DQ0602 [47, 61].

\subsection{Immunopathology of Narcolepsy}

Current evidence on the pathogenesis of narcolepsy comes from studies being focused on type 1 narcolepsy that indicates clearly the involvement of both genetic and environmental factors [10]. Taking into account the immunological features of narcolepsy: (HLA-DQB $1 * 0602$ association, polymorphisms in OX40L, and in the TCR- $\alpha$ locus), death of hypocretin neurons in narcolepsy could be immune mediated (see figure 1) $[8,26,32,39]$. The other replicated evidence for the autoimmune nature of narcolepsy is the recent identification of reactive autoantibodies against the Tribbles 2 homolog (Trib2) protein in sera of narcolepsy patients $[8,28,31,32,39]$. Trib2, a protein expressed in Hcrt cells is an autoantigen in human narcolepsy $[28,32,39]$ and autoimmune uveitis [28]. However, the role of anti Trib2 antibodies in narcolepsy is elusive, either it may kill Hcrt neurons directly or it may be a downstream effect of the cell loss $[28,31,62]$.
DNMT1 is highly expressed in the immune system and plays an important role in differentiation of $\mathrm{CD}^{+} \mathrm{T}$ cells into $\mathrm{T}$ regulatory cells by relieving repression of Foxp3 expression following TCR stimulation. Immunochip study indicates that SNPs in the DNMT1 could alter Treg cell development and/or function and lead to narcolepsy $[2,8]$, besides DNMT1 has effect on survival of hypocretin neurons and neurodegeneration $[2,8,26]$, suggesting the importance of DNA methylation in the pathology [8].

P2RY11 is an ATP receptor which is highly expressed in $\mathrm{CD}^{+} \mathrm{T}$ lymphocytes and natural killer (NK) cells $[8,39]$, and modulates leukocyte maturation, chemotaxis, and apoptosis [2, 35]. SNP in the $3^{\prime}$ untranslated region of P2RY11 causes low expression of P2RY11 and could lead to reduced resistance to ATP-mediated cell death, potentially altering the immune response to infection or directly effecting hypocretin secreting neurons [2].

Cathepsin $\mathrm{H}$ is an endosomal/lysosomal enzyme involved in numerous cellular process including, antigen processing and peptide-MHC II interactions, so SNPs in CTSH could alter the MHC II-peptide repertoire presented to T cells and lead to narcolepsy [2]. OX40L is an important co-stimulatory molecule that functions to promote $\mathrm{T}$ cell survival, expansion, and effector functions [8]. Like CTSH, OX40L is primarily expressed in MHC Class II positive APCs $[2,26]$. OX40 signaling within responding $\mathrm{T}$ cells renders them resistant to Treg mediated suppression, and acts within the Treg cells to inhibit suppressive functions; moreover, excessive OX40-OX40L signaling may sustain inflammatory response and increase survival of effector T-cells that may target hypocretin neurons [26]. 


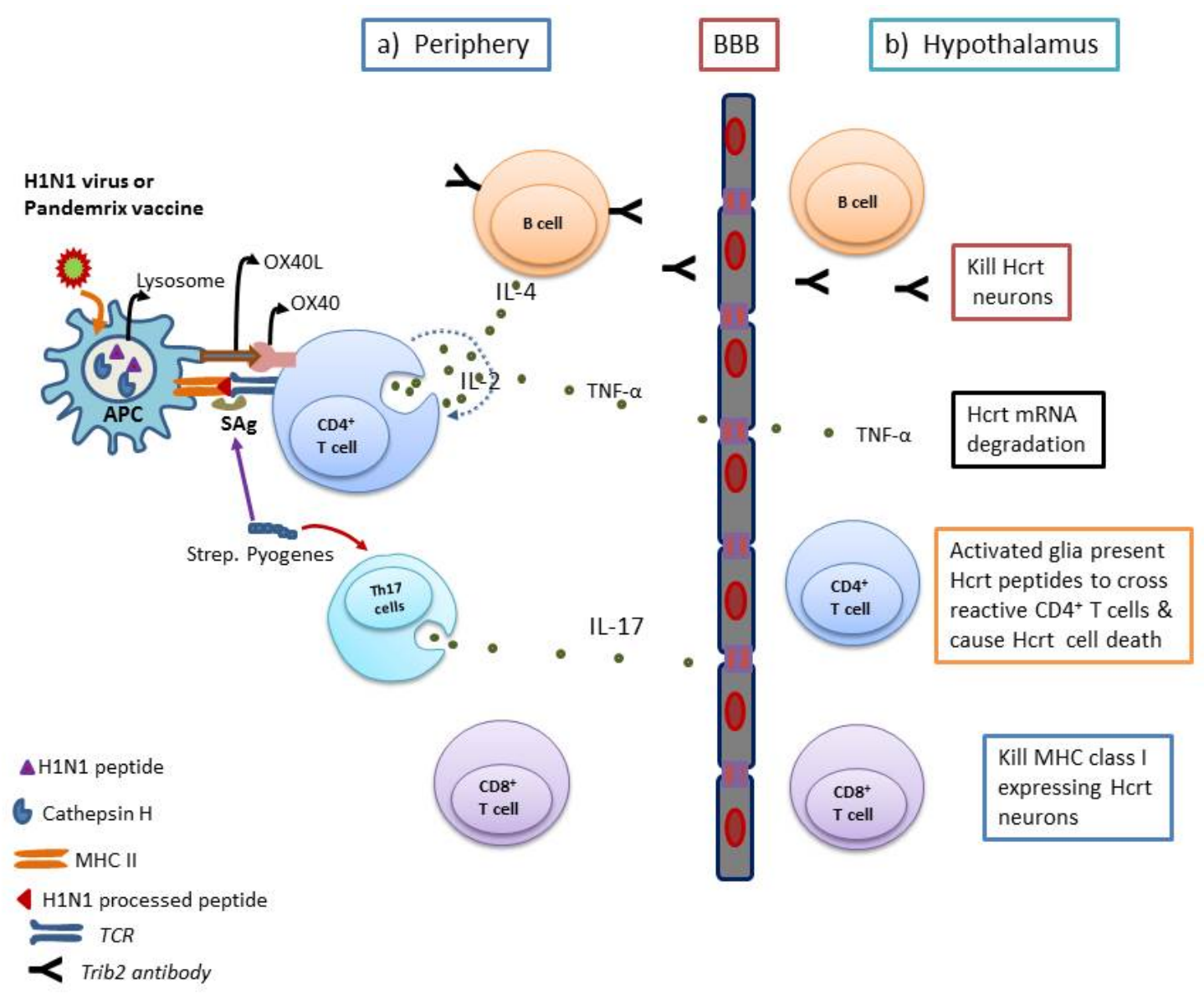

Figure 1. Autoimmune mediated destruction of hypocretin cell in narcolepsy

Immune mediated Hcrt loss is a result of a cascade of reactions leading to the activation of MHC class II (DQA1*01:02-DQB1*06:02) positive APCs, $\mathrm{CD}^{+} \mathrm{T}$ cells, $\mathrm{CD} 8^{+} \mathrm{T}$ cells and $\mathrm{B}$ cells which are reactive to both $\mathrm{H} 1 \mathrm{~N} 1$ influenza virus \& Hcrt neurons. After recognition, APCs prime $\mathrm{CD}^{+} \mathrm{T}$ cells, activated $\mathrm{CD} 4^{+} \mathrm{T}$ cells secrete IFN-g, $\mathrm{TNF} \alpha$ and IL-2, which stimulate $\mathrm{CD} 8^{+} \mathrm{T}$ cells and drive a Th1 mediated immune response. Moreover, TNF $\alpha$ can impair the Hcrt system through degradation of Hcrt mRNA and protein ubiquitination [63]. IL-4 from activated $\mathrm{CD}^{+} \mathrm{T}$ cells promotes a humoral response leading to the production of Tribbles 2 antibody by B cells. $\mathrm{CD} 4^{+} \mathrm{T}$ cells differentiate into Th17 cells that can produce IL-17, and IL-17 signaling enhances permeability of the BBB and neuroinflammation. Figure modified from [2].

\section{Conclusions}

The strong genetic association of narcolepsy with HLA-DQB1*06:02 and SNPs in the TCR $\alpha, \mathrm{P} 2 \mathrm{RY} 11$, DNMT1, CTSH and TNFSF4, and upper airway infections is consistent to be T-cell mediated an autoimmune process $[2,8$, $18,26]$. Moreover, infection with influenza A H1N1 strain or AS03 adjuvanted $\mathrm{H} 1 \mathrm{~N} 1$ vaccine has been reported to cause a cross reactive $\mathrm{CD} 4^{+} \mathrm{T}$ cell response. Whereas streptococcus infection affects BBB integrity and predisposed the immune privileged CNS to auto reactive $\mathrm{T}$ cells, then subsequently caused destruction of the Hcrt neurons in the lateral hypothalamus. Nevertheless, superantigens interact with beta chain of TCR and alpha chain of MHC class II, but this interaction in narcolepsy is reversed. So, a detailed understanding of the nature of this interaction is essential in narcolepsy for understanding the effect of superantigens on $\mathrm{T}$ cells, and their activation and for designing therapeutic strategies by modulating immune cells. There are still some subjects of H1N1 vaccine that remain to be established. Why other H1N1 vaccines, such as those with squalene alone as an adjuvant (MF59 adjuvanted) or non-adjuvanted vaccines didn't induce narcolepsy remains unclear. Progress is needed to better understand the precise T-cell mediated autoimmune process in $\mathrm{AS} 03$ adjuvanted $\mathrm{H} 1 \mathrm{~N} 1$ vaccine induced autoimmunity, and Streptococcus Pyogenes superantigens induced bystander activation of $\mathrm{T}$ cells in narcolepsy. As MHC class II - Streptococcus Pyogenes superantigens TCR interaction in narcolepsy is deviated from the usual way of interaction. Such knowledge will allow researchers to uncover mechanisms to harness the immune response for maximum host protection with minimum damage. 


\section{Acknowledgements}

This is not an organization funded work.

\section{Disclosures}

The authors declare no conflict of interest.

\section{REFERENCES}

[1] De la Herrán-Arita, A.K. and F. García-García, Narcolepsy as an immune-mediated disease. Sleep disorders, 2014.

[2] Mahlios, J., A.K. De la Herrán-Arita, and E. Mignot, The autoimmune basis of narcolepsy. Current opinion in neurobiology, 2013. 23(5): p. 767-773.

[3] Partinen, M., et al., Narcolepsy as an autoimmune disease: the role of H1N1 infection and vaccination. The Lancet Neurology, 2014. 13(6): p. 600-613.

[4] Bergman, P., et al., Narcolepsy patients have antibodies that stain distinct cell populations in rat brain and influence sleep patterns. Proceedings of the National Academy of Sciences, 2014. 111(35): p. E3735-E3744.

[5] Ebrahim, I., et al., Hypocretin (orexin) deficiency in narcolepsy and primary hypersomnia. Journal of Neurology, Neurosurgery \& Psychiatry, 2003. 74(1): p. 127-130.

[6] Plazzi, G., et al., Complex movement disorders at disease onset in childhood narcolepsy with cataplexy. Brain, 2011. 134(12): p. 3480-3492.

[7] Montplaisir, J., et al., Risk of narcolepsy associated with inactivated adjuvanted (AS03) A/H1N1 (2009) pandemic influenza vaccine in Quebec. PloS one, 2014. 9(9): p. e108489.

[8] Singh, A.K., J. Mahlios, and E. Mignot, Genetic association, seasonal infections and autoimmune basis of narcolepsy. Journal of autoimmunity, 2013. 43: p. 26-31.

[9] Costa, F.D., et al., Narcolepsy in pediatric age-Experience of a tertiary pediatric hospital. Sleep Science, 2014. 7(1): p. 53-58.

[10] Kumar, S. and H. Sagili, Etiopathogenesis and Neurobiology of Narcolepsy: A review. Journal of clinical and diagnostic research: JCDR, 2014. 8(2): p. 190.

[11] Partinen, M., et al., Increased incidence and clinical picture of childhood narcolepsy following the 2009 H1N1 pandemic vaccination campaign in Finland. PloS one, 2012. 7(3): p. e33723.

[12] Han, F., Sleepiness that cannot be overcome: narcolepsy and cataplexy. Respirology, 2012. 17(8): p. 1157-1165.

[13] Akintomide, G.S. and H. Rickards, Narcolepsy: a review. Neuropsychiatric disease and treatment, 2011. 7: p. 507.

[14] Nishino, S., Clinical and neurobiological aspects of narcolepsy. Sleep medicine, 2007. 8(4): p. 373-399.
[15] Overeem, S., J.L. Black, and G.J. Lammers, Narcolepsy: immunological aspects. Sleep medicine reviews, 2008. 12(2): p. 95-107.

[16] Nohynek, H., et al., AS03 adjuvanted AH1N1 vaccine associated with an abrupt increase in the incidence of childhood narcolepsy in Finland. PloS one, 2012. 7(3): p. e33536.

[17] Kornum, B.R., J. Faraco, and E. Mignot, Narcolepsy with hypocretin/orexin deficiency, infections and autoimmunity of the brain. Current opinion in neurobiology, 2011. 21(6): p. 897-903.

[18] Han, F., et al., Genome wide analysis of narcolepsy in China implicates novel immune loci and reveals changes in association prior to versus after the 2009 H1N1 influenza pandemic. PLoS genetics, 2013. 9(10): p. e1003880.

[19] Partinen, M., et al., Does autoreactivity have a role in narcolepsy? The Lancet Neurology, 2014. 13(11): p. 1072-1073.

[20] Bayard, S., et al., Executive control of attention in narcolepsy. PloS one, 2012. 7(4): p. e33525.

[21] Holm, A., et al., miRNA profiles in plasma from patients with sleep disorders reveal dysregulation of miRNAs in narcolepsy and other central hypersomnias. Sleep, 2014. 37(9): p. 1525.

[22] Juji, T., M. Satake, and Y. Honda, HLA antigens in Japanese patients with narcolepsy. Tissue antigens, 1984. 24(5): p. 316-319.

[23] Mignot, E., Genetics of narcolepsy and other sleep disorders. The American Journal of Human Genetics, 1997. 60(6): p. 1289-1302.

[24] Siebold, C., et al., Crystal structure of HLA-DQ0602 that protects against type 1 diabetes and confers strong susceptibility to narcolepsy. Proceedings of the National Academy of Sciences of the United States of America, 2004. 101(7): p. 1999-2004.

[25] Parish, J.M., Genetic and immunologic aspects of sleep and sleep disorders. CHEST Journal, 2013. 143(5): p. 1489-1499.

[26] Faraco, J., et al., ImmunoChip study implicates antigen presentation to T cells in narcolepsy. PLoS genetics, 2013. 9(2): p. e1003270.

[27] Caillat - Zucman, S., Molecular mechanisms of HLA association with autoimmune diseases. Tissue antigens, 2009. 73(1): p. $1-8$.

[28] Cvetkovic-Lopes, V., et al., Elevated Tribbles homolog 2specific antibody levels in narcolepsy patients. The Journal of clinical investigation, 2010. 120(3): p. 713.

[29] Nishino, S., et al., Hypocretin/orexin and narcolepsy: new basic and clinical insights. Acta physiologica, 2010. 198(3): p. 209-222.

[30] Watson, N.F., et al., Does narcolepsy symptom severity vary according to HLA-DQB1*0602 allele status? Sleep, 2010. 33(1): p. 29.

[31] Faraco, J. and E. Mignot, Immunological and genetic aspects of narcolepsy. Sleep Medicine Research (SMR), 2011. 2(2): p. 39-47. 
[32] Fontana, A., et al., Narcolepsy: autoimmunity, effector T cell activation due to infection, or $T$ cell independent, major histocompatibility complex class II induced neuronal loss? Brain, 2010: p. awq086.

[33] Goel, N., et al., $D Q B 1^{*} 0602$ predicts interindividual differences in physiologic sleep, sleepiness, and fatigue. Neurology, 2010. 75(17): p. 1509-1519.

[34] Koepsell, T.D., W.T. Longstreth, and T.G. Ton, Medical exposures in youth and the frequency of narcolepsy with cataplexy: a population - based case-control study in genetically predisposed people. Journal of sleep research, 2010. 19 (1 - Part - I): p. 80-86.

[35] Han, F., et al., TCRA, P2RY11, and CPT1B/CHKB associations in Chinese narcolepsy. Sleep medicine, 2012. 13(3): p. 269-272.

[36] Fontana, A., H. Gast, and T. Birchler, Narcolepsy: Autoimmunity or Secondary to Infection?, in Narcolepsy2011, Springer. p. 19-26.

[37] Hallmayer, J., et al., Narcolepsy is strongly associated with the T-cell receptor alpha locus. Nature genetics, 2009. 41(6): p. 708-711.

[38] Ambati, A., et al., Increased $\beta$ - haemolytic group $A$ streptococcal M6 serotype and streptodornase $B$ - specific cellular immune responses in Swedish narcolepsy cases. Journal of internal medicine, 2015.

[39] Kornum, B.R., et al., Common variants in P2RY11 are associated with narcolepsy. Nature genetics, 2011. 43(1): p. 66-71.

[40] Dauvilliers, Y., et al., Increased risk of narcolepsy in children and adults after pandemic H1N1 vaccination in France. Brain, 2013. 136(8): p. 2486-2496

[41] Coelho, F., et al., TNF and IL-6 levels in narcoleptic patients. Einstein, 2006. 4(4).

[42] Dauvilliers, Y., et al., Post-H1N1 narcolepsy-cataplexy. Sleep, 2010. 33(11): p. 1428.

[43] Chen, L., et al., Animal models of narcolepsy. CNS \& neurological disorders drug targets, 2009. 8(4): p. 296.

[44] Mignot, E., Sleep, sleep disorders and hypocretin (orexin). Sleep medicine, 2004. 5: p. S2-S8.

[45] Sfriso, P., et al., Infections and autoimmunity: the multifaceted relationship. Journal of leukocyte biology, 2010. 87(3): p. 385-395.

[46] Miller, E., et al., Risk of narcolepsy in children and young people receiving ASO3 adjuvanted pandemic A/H1N1 2009 influenza vaccine: retrospective analysis. BMJ, 2013. 346: p. f794.

[47] Alberto, K., et al., CD4+ $T$ cell autoimmunity to hypocretin/orexin and cross-reactivity to a 2009 H1N1 influenza A epitope in narcolepsy. Science translational medicine, 2013. 5(216): p. 216ra176-216ra176.

[48] Han, F., et al., Narcolepsy onset is seasonal and increased following the 2009 H1N1 pandemic in China. Annals of neurology, 2011. 70(3): p. 410-417.
[49] Ercolini, A. and S. Miller, The role of infections in autoimmune disease. Clinical \& Experimental Immunology, 2009. 155(1): p. 1-15.

[50] Mignot, E., Y. Dauvilliers, and J. Montplaisir, Comment on the Letter to the Editor by Dr. Marcus on the association between narcolepsy and H1N1 exposure. Sleep, 2011. 34(6): p. 689.

[51] Mendes, M.F.S.G., et al., Narcolepsy after A/H1N1 vaccination. Clinics, 2012. 67(1): p. 77-78.

[52] Longstreth Jr, W., T.G. Ton, and T.D. Koepsell, Narcolepsy and streptococcal infections. Sleep, 2009. 32(12): p. 1548.

[53] Natarajan, N., et al., Narcolepsy-cataplexy: is streptococcal infection a trigger? Journal of clinical sleep medicine: JCSM: official publication of the American Academy of Sleep Medicine, 2013. 9(3): p. 269.

[54] Aran, A., et al., Elevated anti-streptococcal antibodies in patients with recent narcolepsy onset. Sleep, 2009. 32(8): p. 979.

[55] Ahmed, S.S., et al., Narcolepsy, 2009 A (H1N1) pandemic influenza, and pandemic influenza vaccinations: What is known and unknown about the neurological disorder, the role for autoimmunity, and vaccine adjuvants. Journal of autoimmunity, 2014. 50: p. 1-11.

[56] Bardage, C., et al., Neurological and autoimmune disorders after vaccination against pandemic influenza A (H1N1) with a monovalent adjuvanted vaccine: population based cohort study in Stockholm, Sweden. BMJ, 2011. 343.

[57] Harris, T., et al., Did narcolepsy occur following administration of ASO3-adjuvanted $A$ (H1N1) pandemic vaccine in Ontario, Canada? A review of post-marketing safety surveillance data. Euro surveillance: bulletin Européen sur les maladies transmissibles= European communicable disease bulletin, 2014. 19(36).

[58] Winstone, A.M., et al., Clinical features of narcolepsy in children vaccinated with ASO3 adjuvanted pandemic A/H1N1 2009 influenza vaccine in England. Developmental Medicine \& Child Neurology, 2014. 56(11): p. 1117-1123.

[59] Melen, K., et al., No serological evidence of influenza $A$ H1N1pdm09 virus infection as a contributing factor in childhood narcolepsy after Pandemrix vaccination campaign in Finland. PloS one, 2013. 8(8): p. e68402.

[60] Masoudi, S., et al., The adjuvant component $\alpha$-tocopherol triggers via modulation of Nrf2 the expression and turnover of hypocretin in vitro and its implication to the development of narcolepsy. Vaccine, 2014. 32(25): p. 2980-2988.

[61] Wucherpfennig, K.W., Mechanisms for the induction of autoimmunity by infectious agents. Journal of Clinical Investigation, 2001. 108(8): p. 1097.

[62] Lim, A.S. and T.E. Scammell, The trouble with Tribbles: do antibodies against TRIB2 cause narcolepsy? Sleep, 2010. 33(7): p. 857.

[63] Zhan, S., et al., Tumor necrosis factor-alpha regulates the Hypocretin system via $m R N A$ degradation and ubiquitination. Biochimica et Biophysica Acta (BBA)-Molecular Basis of Disease, 2011. 1812(4): p. 565-571. 